\section{Palpitations in a 64-year-old}

\section{man}

\section{ABSTRACT}

A 64-year-old man presented with several weeks of intermittent irregular palpitations. He had no prior history of cardiac disease, hypertension or syncope. A 12-lead ECG revealed sinus rhythm with premature atrial and ventricular contractions and high QRS voltages consistent with LV-hypertrophy. Cardiac MR revealed asymmetrical septal hypertrophy and marked mid-myocardial hyperenhancement of the interventricular septum.

A 64-year-old man presented with several weeks of intermittent irregular palpitations. He had no prior history of cardiac disease, hypertension or syncope. A 12-lead ECG revealed sinus rhythm with premature atrial and ventricular contractions and high QRS voltages consistent with LV-hypertrophy (figure 1).
Echocardiography showed asymmetrical septal hypertrophy but image quality was very limited due to poor acoustic windows. Cardiac MR was performed to better visualise myocardial morphology. This revealed asymmetrical septal hypertrophy $(2.3 \mathrm{~cm}$ septum vs $1.0 \mathrm{~cm}$ inferolateral wall) (white arrow figure $2 \mathrm{~A}, \mathrm{~B}$ ) with a mildly enlarged LV cavity and an EF of $42 \%$ (see online supplementary videos 1 and 2). Late gadolinium enhancement imaging showed marked mid-myocardial hyperenhancement of the interventricular septum (figure 2C,D).

\section{QUESTION}

The most appropriate next step is:

A. Coronary angiography

B. Implantable defibrillator placement

C. Endomyocardial biopsy

D. Invasive electrophysiology study

For answers see page 177

Heart 2014;100:167-168. doi:10.1136/heartjnl-2013-305096

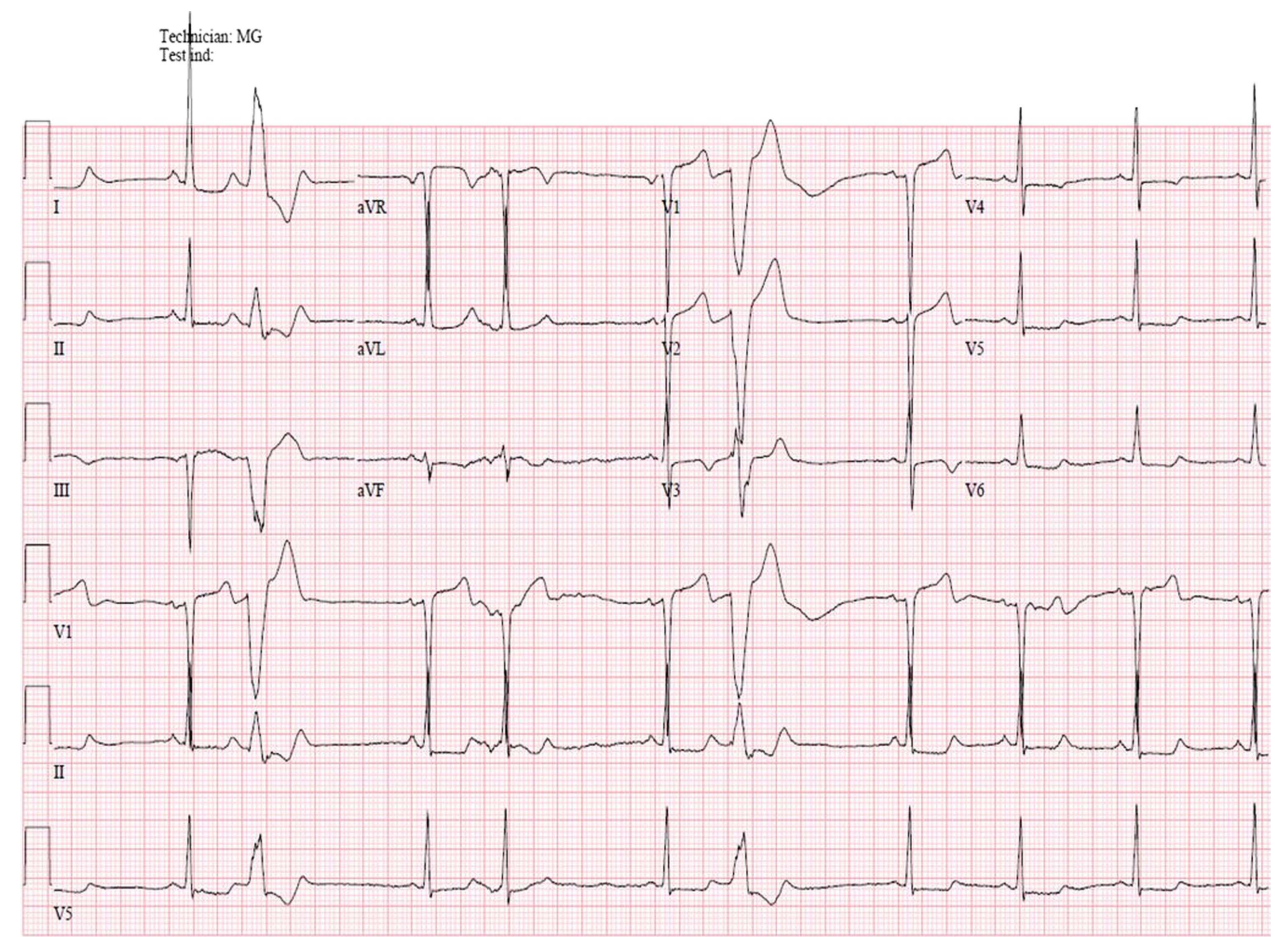

Figure 1 12-lead ECG showing sinus rhythm with premature atrial and ventricular contractions and high QRS voltages consistent with LV-hypertrophy. 
Figure 2 (A) Short-axis cine image showing asymmetrical septal hypertrophy $(2.3 \mathrm{~cm}$ septum (white arrow) vs $1.0 \mathrm{~cm}$ inferolateral wall) with a mildly enlarged LV cavity. (B) Four-chamber cine image showing asymmetrical septal hypertrophy ( $2.3 \mathrm{~cm}$ septum (white arrows) vs enlarged LV cavity. (C) Short-axis image showing marked mid-myocardial hyperenhancement of the interventricular septum. (D)

Four-chamber image showing marked mid-myocardial hyperenhancement of the interventricular septum. $1.0 \mathrm{~cm}$ inferolateral wall) with a mildly

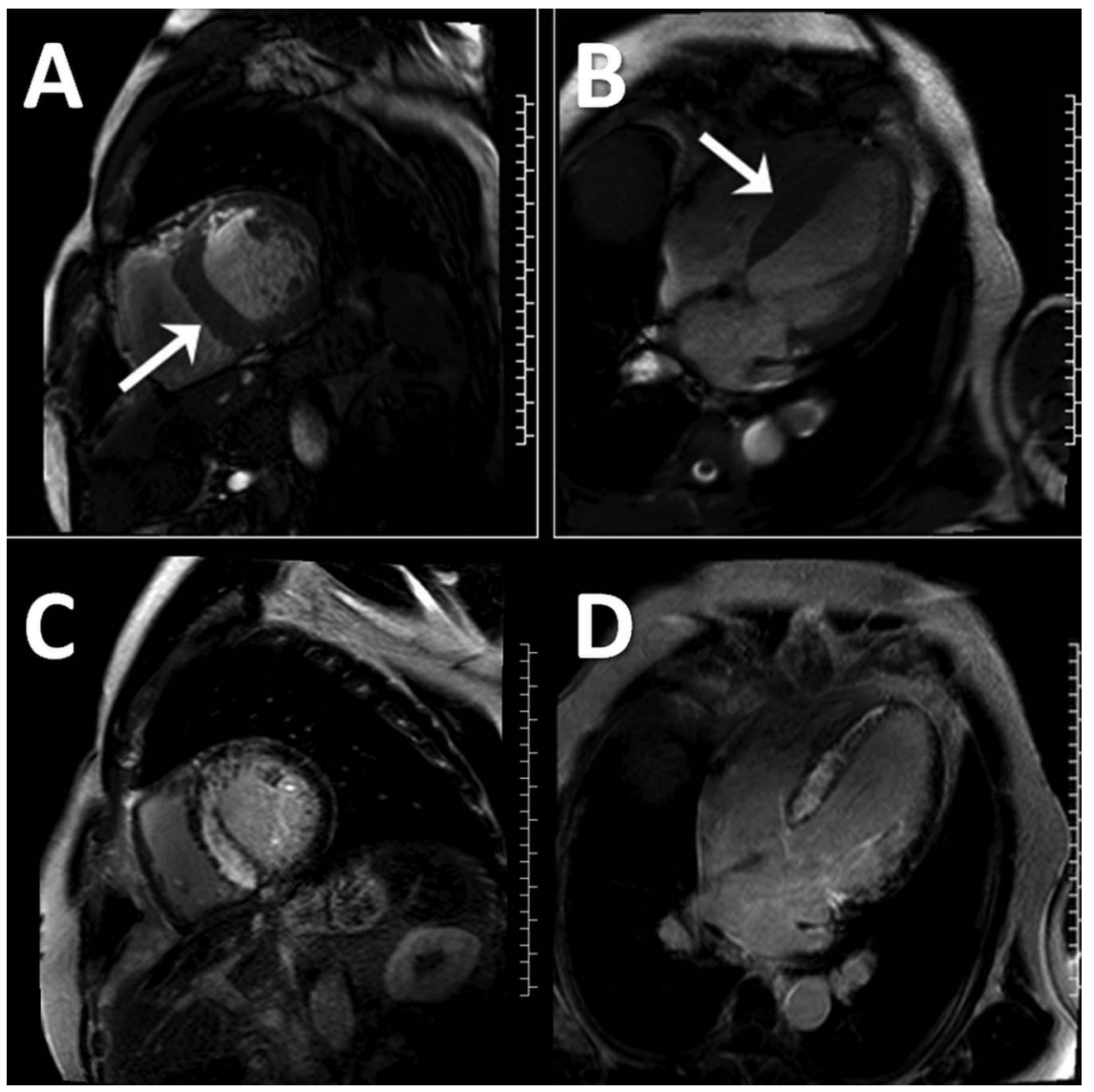

Figure 3 (A) Endomyocardial biopsy showing amyloid deposition on $\mathrm{H} \& \mathrm{E}$ staining. (B) Endomyocardial biopsy showing amyloid deposition on Congo red staining.

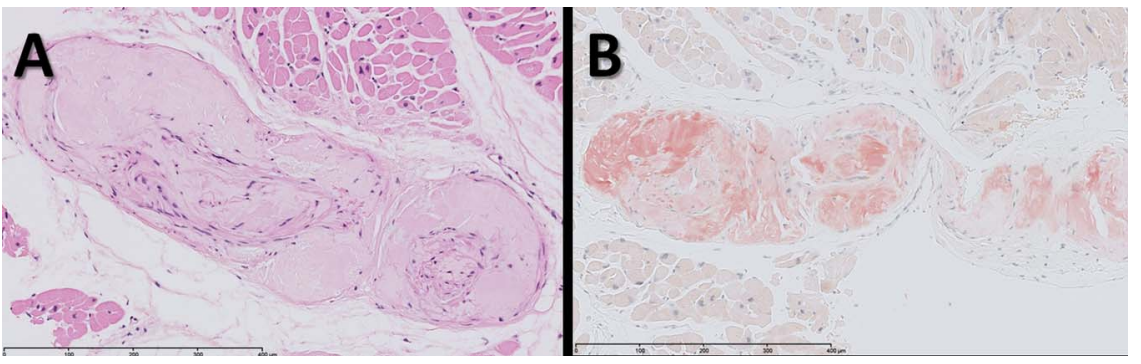




\section{IMAGE CHALLENGE}

\section{Palpitations in a 64-year-old man}

\section{ANSWER}

From question on page 167

C: In myocardial infarction, hyperenhancement invariably involves the subendocardium, which is spared in this case. The mid-wall hyperenhancement seen here is highly suggestive of a non-coronary artery disease aetiology. Mid-myocardial septal hyperenhancement in association with asymmetrical septal hypertrophy is common in hypertrophic cardiomyopathy. ${ }^{1} 2$ However, the hyperenhancement characteristically appears in a diffuse, patchy mid-wall distribution with predilection for the anterior and posterior RV insertion sites. In this case, the extent and intensity of the hyperenhancement as well as the presence of LV enlargement and dysfunction were not typical for hypertrophic cardiomyopathy. There is no indication for implantable defibrillator placement or invasive electrophysiology study at this stage. Endomyocardial biopsy was therefore performed to try to obtain a diagnosis. This showed amyloid deposition on $\mathrm{H} \& \mathrm{E}$ (figure $3 \mathrm{~A}$ ) as well as Congo red (figure 3B) staining confirming the diagnosis of cardiac amyloid. Light-chain amyloidosis was confirmed on further workup including bone marrow biopsy.

This case demonstrates that cardiac amyloid can occasionally present with electrocardiographic LV-hypertrophy, asymmetrical septal hypertrophy and a focal mid-myocardial (as opposed to diffuse subendocardial) hyperenhancement pattern on cardiac MR, which may mimic hypertrophic cardiomyopathy. ${ }^{1-4}$ Distinguishing between these entities may require endomyocardial biopsy and has significant prognostic and therapeutic implications.
Michael A Bauml, ${ }^{1}$ Jaehoon Chung, ${ }^{1}$ John V Groth, ${ }^{2}$ Afshin Farzaneh-Far ${ }^{1,3,4}$

${ }^{1}$ Section of Cardiology, Department of Medicine, University of Illinois at Chicago, Chicago, Illinois, USA

${ }^{2}$ Department of Pathology, University of Illinois at Chicago, Chicago, Illinois, USA

${ }^{3}$ Department of Radiology, University of Illinois at Chicago, Chicago, Illinois, USA

${ }^{4}$ Division of Cardiology, Department of Medicine, Duke University, Durham, North

Carolina, USA

Correspondence to Dr Afshin Farzaneh-Far, Section of Cardiology, Department of Medicine, University of Illinois at Chicago, 840 South Wood St, M/C 715, Suite 920

S, Chicago, IL 60612, USA; afshin@uic.edu

- Additional material is published online only. To view please visit the journal online (http://dx.doi.org/10.1136/heartjnl-2013-305096).

Contributors AF-F cared for the patient, supervised all imaging studies and drafted the manuscript. MAB helped draft the manuscript. All authors contributed to writing and editing of manuscript. All authors read and approved the final manuscript.

Competing interests None.

Patient consent Obtained.

Ethics approval Institutional IRB.

Provenance and peer review Not commissioned; internally peer reviewed.

To cite Bauml MA, Chung J, Groth JV, et al. Heart 2014;100:177.

Received 14 October 2013

Revised 19 October 2013

Accepted 21 October 2013

Published Online First 26 November 2013

Heart 2014;100:177. doi:10.1136/heartjnl-2013-305096a

\section{REFERENCES}

1 Choudhury L, Mahrholdt H, Wagner A, et al. Myocardial scarring in asymptomatic or mildly symptomatic patients with hypertrophic cardiomyopathy. J Am Coll Cardiol 2002;40:2156-64.

2 Moon JC, McKenna WJ, McCrohan JA, et al. Toward clinical risk assessment in hypertrophic cardiomyopathy with gadolinium cardiovascular magnetic resonance. J Am Coll Cardiol 2003;41:1561-7.

3 Maceira AM, Joshi J, Prasad SK, et al. Cardiovascular magnetic resonance in cardiac amyloidosis. Circulation 2005;111:186-93.

4 Syed IS, Glockner JF, Feng D, et al. Role of cardiac magnetic resonance imaging in the detection of cardiac amyloidosis. JACC Cardiovasc Imaging 2010;3:155-64. 